\title{
Different glucose metabolism behavior relating to histotypes in synchronous breast cancers evaluated by $\left[{ }^{18 F}\right]$ FDG PET-CT
}

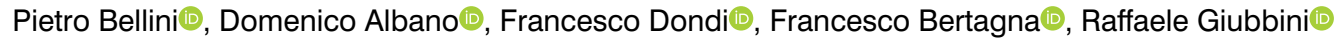
Nuclear Medicine, University of Brescia and ASST Spedali Civili Brescia, Brescia, Italy

[Received 07 IV 2021; Accepted 12 X 2021]

\begin{abstract}
Glucose metabolism is increased in most aggressive tumors and it is commonly evaluated by positron emission tomography-computed tomography (PET-CT) with ${ }^{18} \mathrm{~F}$-fluorodeoxyglucose ([18 F]FDG), measuring the Maximum Standardized Uptake Value (SUVmax) for the assessment. Particularly, it is known that breast cancer expresses different glucose metabolism in relation to estrogen receptor (ER), progesterone receptor (PR), Ki67 score, tumor grading, tumor size, and Human Epidermal Growth Factor Receptor 2 (HER2). We present an interesting case of a woman with two different, synchronous breast cancers characterized by different glucose metabolism, according to literature knowledge.
\end{abstract}

KEY words: breast cancer; $\left[{ }^{18} \mathrm{~F}\right] \mathrm{FDG}$; PET; histotype; glucose metabolism

Nucl Med Rev 2022; 25, 1: 64-65

During breast cancer screening performed with ultrasonography and mammography, in a 91-years-old woman two bilateral breast nodules were found and both nodules appeared oncologically suspicious. Trucut biopsy was subsequently performed on both lesions, with the confirmation of their malignancy.

The left lesion was diagnosed as invasive breast cancer, no special histotype, grading stage G3 with a 33\% Ki67 expression, positive for estrogen receptor (ER), negative for progesterone receptor (PR), and positive for Human Epidermal Growth Factor Receptor 2 (HER2).

In contrast, the right lesion was diagnosed as invasive breast cancer, however with unclear histotype, suspicious for lobular carcinoma, grading stage G2 with a 20\% Ki67 expression, positive for ER and PR, and without HER2 expression.

Correspondence to: Pietro Bellini, Nuclear Medicine,

Spedali Civili di Brescia, P.le Spedali Civili, 125123 Brescia Italy,

tel.: +39-30-3995461, fax: +39-30-3995420

e-mail: bellini.pietro@outlook.it
Then ${ }^{18} \mathrm{~F}$-fluorodeoxyglucose positron emission tomography-computed tomography ([18F]FDG PET-CT) was performed for staging purposes.

The tumor size of the right lesion was $23 \mathrm{~mm} \times 19 \mathrm{~mm} \times 19 \mathrm{~mm}$ and FDG uptake was moderate with a Maximum Standardized Uptake Value (SUVmax) of 5.82 and a Metabolic Tumor Volume (MTV) of $7.1 \mathrm{~cm}^{3}$ (Fig. 1).

The tumor size of the left lesion was $25 \mathrm{~mm} \times 19 \mathrm{~mm} \times 20 \mathrm{~mm}$, with MTV of $7.6 \mathrm{~cm}^{3}$ and SUVmax of 16.05 (Fig. 2).

The two breast lesions had similar tumor size and MTV values (MTV ratio between the two was 1.07), but a significantly different FDG uptake, expressed as SUVmax (SUVmax ratio between the two was 2.76)

Due to the age of the patient, no surgery was performed and Letrozole therapy was started.

It is known that SUVmax in breast cancer lesions has a positive correlation with Ki67 expression [1, 2] and the grading of the lesion [1]. A similar positive correlation with nodule size [1], presence of HER2 [1], and absence of PR [1, 2] was also suggested by other studies, however without shared consensus. 


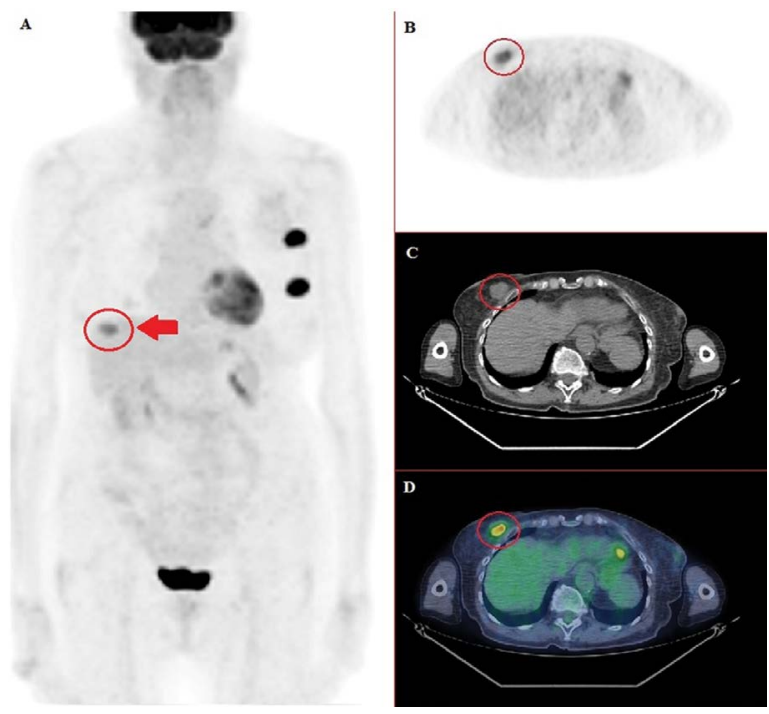

Figure 1. Maximum Intensity Projection (MIP) (A), axial PET (B), axial CT (C) and fused PET-CT in FRENCH color (D) images of a PET-CT scan reporting bilateral breast lesions (right lesion labeled by red circle and arrow) and left axillary metastasis
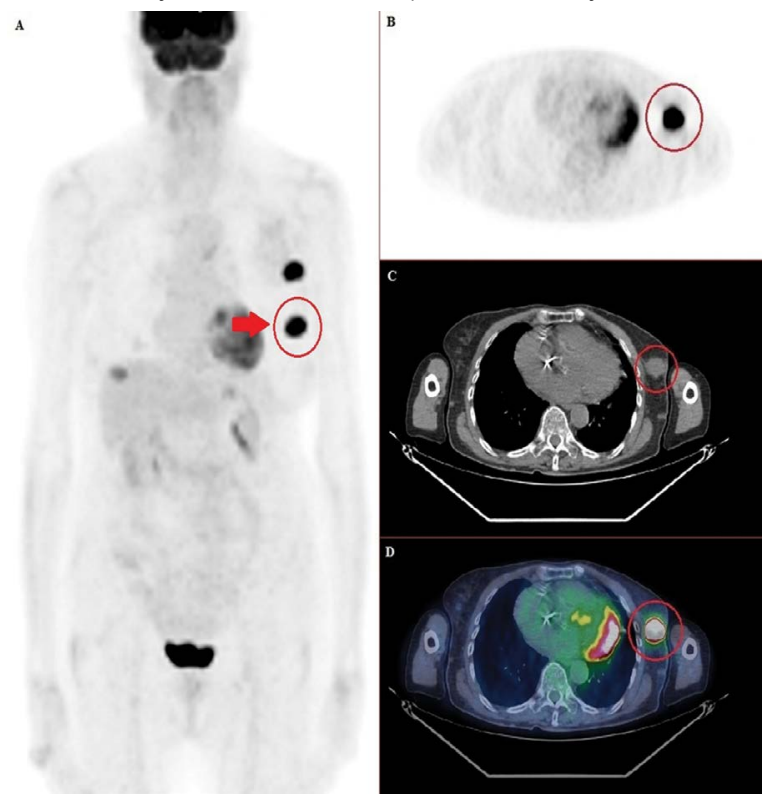

Figure 2. MIP (A), axial PET (B), axial CT (C) and fused PET-CT in FRENCH color (D) images of the same PET-CT scan (left lesion labeled by red circle and arrow)

According to the literature, our case report underlines two metabolic patterns related to different biological and genetic characteristics of the breast lesions with similar size in the same patient, in particular without the presence of confounding volume difference.

Bilateral breast cancer is an uncommon finding [3], even more, if presented with different metabolic behavior as reported and clearly showed by $\left[{ }^{18} \mathrm{~F}\right] \mathrm{FDG}$ PET-CT.

\section{Conflict of interest}

The authors declare that they have no conflict of interest.

\section{References}

1. Cerci SM, Bozkurt KK, Eroglu HE, et al. Evaluation of the association between HIF-1 and HER-2 expression, hormone receptor status, Ki-67 expression, histology and tumor FDG uptake in breast cancer. Oncol Lett. 2016; 12(5): 3889-3895, doi: 10.3892/ol.2016.5199, indexed in Pubmed: 27895745.

2. Qu YH, Long N, Ran C, et al. The correlation of F-FDG PET/CT metabolic parameters, clinicopathological factors, and prognosis in breast cancer. Clin TransI Oncol. 2021; 23(3): 620-627, doi: 10.1007/s12094-020-02457-w, indexed in Pubmed: 32683540.

3. Albano D, Bertoli M, Morassi ML, et al. Incidental 18F-FDG PET/CT bilateral breast uptake due to carcinoma. Nucl Med Rev Cent East Eur. 2016; 19(B): 14-16, doi: 10.5603/NMR.2016.0028, indexed in Pubmed: 27900755. 